\title{
A ROLE FOR CXCR4 IN PERITONEAL AND HEMATOGENOUS OVARIAN CANCER DISSEMINATION
}

Agnès Figueras ${ }^{1,7}$, Elisenda Alsina-Sanchís ${ }^{1,7}$, Álvaro Lahiguera ${ }^{1,7}$, Manuel Abreu $^{2}$, Laura Muinelo-Romay ${ }^{2}$, Gema Moreno-Bueno ${ }^{3,4}$, O. Casanovas ${ }^{1,7}, \mathrm{M}$. Graupera $^{1,5,7}$, Xavier Matias-Guiu ${ }^{6,7}$, August Vidal ${ }^{6,7,8,9}$, Alberto Villanueva ${ }^{1,7,8}$, Francesc Viñals ${ }^{1,7,10^{*}}$

1 Program Against Cancer Therapeutic Resistance (ProCURE), Institut Català d'Oncologia, Hospital Duran i Reynals, 08908 L'Hospitalet de Llobregat, Barcelona, Spain; 2 Liquid Biopsy Analysis Unit, Oncomet, Health Research Institute of Santiago de Compostela (IDIS), CIBERONC, Santiago de Compostela, Spain; 3 Departamento de Bioquímica, Universidad Autónoma de Madrid (UAM), Instituto de Investigaciones Biomédicas "Alberto Sols" (CSIC-UAM), IdiPaz, CIBERONC, Madrid, Spain; 4 MD Anderson International Foundation, Madrid, Spain; 5 Laboratori d'Oncologia Molecular, Institut d'Investigació Biomèdica de Bellvitge (IDIBELL), 08908 L'Hospitalet de Llobregat, Barcelona, Spain; 6 Servei d'Anatomia Patològica, Hospital Universitari de Bellvitge, CIBERONC, 08908 L'Hospitalet de Llobregat, Barcelona, Spain; 7 Institut d'Investigació Biomèdica de Bellvitge (IDIBELL), 08908 L'Hospitalet de Llobregat, Barcelona, Spain; 8 Xenopat, Carrer de la Feixa Llarga S/N, L'Hospitalet de Llobregat, 
08907 Barcelona, Spain; 9 Departament de Patologia i Terapèutica Experimental and 10 Departament de Ciències Fisiològiques, Universitat de Barcelona, 08908 L'Hospitalet de Llobregat, Barcelona, Spain.

* To whom correspondence should be addressed. Mailing address: Dr. Francesc Viñals - Laboratori de Recerca Translacional, Program Against Cancer Therapeutic Resistance (ProCURE), Institut Català d'Oncologia (IDIBELL), Hospital Duran i Reynals, Gran Via s/n km 2.7, 08908 L'Hospitalet de Llobregat, Barcelona, Spain.

E-mail: fvinyals@iconcologia.net

RUNNING TITLE: CXCR4 induces CTCs in ovarian carcinomas

KEY WORDS: CXCR4, epithelial ovarian carcinoma, EMT, CTCs, metastasis

\section{FINANCIAL SUPPORT}

This study was supported by research grants from the Spanish Ministerio de Economía y Competitividad (SAF2013-46063R), The Spanish Institute of Health Carlos III (ISCIII) and the European Regional Development Fund (ERDF) under the Integrated Project of Excellence no. PIE13/00022 (ONCOPROFILE) and the Generalitat de Catalunya (2014SGR364) to FV. Work supported by the Xarxa de Bancs de Tumors de Catalunya sponsored by Pla Director d'Oncología de Catalunya (XBTC), IDIBELL and PLATAFORMA BIOBANCOS 
PT13/0010/0013. EAS is a recipient of a predoctoral fellowship from the Ministerio de Economía y Competitividad. 


\section{ABSTRACT}

Epithelial ovarian cancer is characterized by a low recovery rate because the disease is typically diagnosed at an advanced stage, by which time, most patients $(80 \%)$ already exhibit disseminated neoplasia. The cytokine receptor CXCR4 has been implicated in the development of metastasis in various tumor types. Using a patient-derived tissue macroarray and mRNA expression analysis we observed high CXCR4 levels in high-grade serous epithelial ovarian carcinomas, the most metastatic tumor, compared with those in endometrioid carcinomas. CXCR4 inhibition by treatment with the CXCR4 antagonist AMD3100 or by expression of shRNA anti-CXCR4 similarly inhibited angiogenesis in several models of ovarian carcinomas orthotopically grown in nude mice, but the effect on tumor growth was correlated with the levels of CXCR4 expression. Moreover, CXCR4 inhibition completely blocked dissemination and metastasis. This effect was associated with reduced levels of active Src, active ERKs, the inhibition of EMT transition and block of hematogenous ovarian cancer dissemination decreasing circulating human tumoral cells (CTCs). In tumors, CXCR4-expressing cells also had more mesenchymal characteristics. In conclusion, our results indicate that CXCR4 expression confers a proinvasive phenotype to ovarian carcinoma cells. Thus anti-CXCR4 therapy is a possible agent for a complementary treatment of advanced disseminated epithelial high-grade serous ovarian cancer patients. 


\section{INTRODUCTION}

Ovarian cancer is the fifth most common cause of cancer deaths in women in western countries $(1,2)$. More than $90 \%$ of malignant ovarian tumors are of epithelial origin and can be classified into five main histological types: high-grade serous (70\% of cases and the most aggressive type), endometrioid (20\% of cases), clear-cell, low-grade serous and mucinous (3). Although progress has been made in treating this cancer by improved surgical debulking and the introduction of platinum-taxane regimens, the overall five-year survival rate is only $42 \%$. This low survival rate is due to diagnosis frequently being made when the disease is at an advanced stage, and to intrinsic and acquired resistance to platinum-based chemotherapy (1).

CXCR4 is a member of the family of chemokine receptors, expressed in different cell types, that mediate cell chemotaxis depending on gradients of chemokines $(4,5)$. CXCR4 belongs to the serpentin family of G-protein-coupled transmembrane receptors. It binds to CXCL12/SDF1 and stimulates various signaling pathways. CXCR4 and CXCL12 are also overexpressed in several cancers, including breast, prostate and ovarian tumors. CXCR4-CXCL12 has been implicated in the migration of cancer cells and the generation of metastasis. In ovarian cancer, CXCR4 expression increases with the stage of ovarian tumors and in metastasis (6-9), while CXCL12 is present in ascites of ovarian cancer patients (10). However, the exact role of the CXCR4-CXCL12 axis in ovarian cancer cell proliferation and metastatic capacity in different ovarian tumors subtypes is still not completely understood. 


\section{MATERIALS AND METHODS}

\section{Chemical compounds}

Lyophilized AMD3100 (octahydrochloride) (11) was obtained from TOCRIS Bioscience (Bristol, UK). It was diluted in sterile serum $(1.5 \mathrm{mg} / \mathrm{ml})$ before intraperitoneal injection. Cell culture media, FBS, glutamine and antibiotics were obtained from Gibco (ThermoFisher Scientific, Waltham, MA). Other reagents were of analytical or molecular biology grade and purchased from Sigma-Aldrich (St Louis, MO).

\section{Patient samples}

Tissue macroarray patient samples: Our retrospective ovarian cancer study population comprised 68 women who were diagnosed with high-grade serous $(n=33)$ or endometrioid $(n=35)$ epithelial ovarian carcinomas resected between 1992 and 2007 at the Hospital of Bellvitge (Barcelona, Spain). The population has been described before (12).

Tumoral RNA patient samples: Our retrospective ovarian cancer study population comprised 12 women (Age=45-79 years) who were diagnosed with high-grade serous $(n=7)$ or endometrioid $(n=6)$ epithelial ovarian carcinomas resected between 2006 and 2016 at the Hospital of Bellvitge (Barcelona, Spain). The majority of the endometrioid samples were classified as T1 FIGO (67\%) and only $33 \%$ of the samples were T3. The serous samples were $100 \%$ T3 FIGO. 
Blood RNA patient samples: 38 ovarian cancer patients (Age=23-83 years) were recruited at MD Anderson Cancer Center (Madrid). Healthy controls $(n=20)$ with an absence of a previous cancer episode and an age matched with patients were also included in the study. $58 \%$ of patients corresponded to high grade serous carcinomas, $8 \%$ to endometrioid and the rest to other subtypes. $5 \%$ corresponded to $\mathrm{T} 1 \mathrm{FIGO}, 13 \%$ to $\mathrm{T} 2$ and $64 \%$ to $\mathrm{T} 3$ or $\mathrm{T} 4$.

All patients signed an informed consent specifically approved by the Ethical Committee corresponding to each institution. All the studies protocols were cleared by the Ethics Committee of each institution and they were conducted in accordance with the World Medical Association Declaration of Helsinki.

\section{Orthotopic implantation of ovarian tumors}

Surgical implantation was performed as described (12). We used orthotopic preclinical models generated after implantation in nude mice. Female nu/nu Swiss mice, strain NMRI-Foxn $1^{n u} / F o x n 1^{n u}$, were purchased from Janvier (Saint Berhevin, France). We implanted tumor samples obtained from untreated patients after surgery (OVAs) or from SKOV-3 cells. The ovarian OVAs used were perpetuated in nude mice by consecutive passages. The study protocol was cleared by the Ethics Committee of Bellvitge Hospital and signed informed consent was obtained from each patient. We chose three ovarian orthotopic models previously generated by our group: two high-grade serous (OVA17 and OVA8) and an endometrioid ovarian tumors (OVA15) (12). 
SKOV-3 cells (bought to Sigma-Aldrich in 2014, passage 5-20, mycoplasma tested by PCR every month) infected with lentivirus expressing GFP-Luciferase (LV-T2A) (13), control shRNA (Sigma MISSION ${ }^{\circledR}$ pLKO.1-pure empty vector, Sigma-Aldrich) or anti-CXCR4 shRNAs (Sigma MISSION ${ }^{\circledR}$ pLKO. 1 lentiviral vectors) were used to permanently silence CXCR4 expression and to generate orthotopic ovarian tumors. As these vectors express puromycin resistance, cell lines expressing lentiviral vectors were established in constant culture of puromycin-containing media $(2 \mu \mathrm{g} / \mathrm{ml})$. Mice were anesthetized with isoflurane and 1 million cells were injected into the ovary in $20 \mu \mathrm{l}$ of DMEM medium.

All the animal studies were approved by the local committee for animal care (IDIBELL, DAAM 5766).

\section{Treatment schedule}

Treatments started when a palpable intra-abdominal mass was detected (1 month after implantation). Studies were terminated when tumors in vehicletreated animals were judged to be adversely affecting their wellbeing.

AMD3100 $(1.5 \mathrm{mg} / \mathrm{ml})$ was administered intraperitoneally every day from Monday to Friday for four weeks. Control group mice received intraperitoneal sterile serum under the same schedule as AMD3100-treated mice. Blood samples were collected from the saphenous vein before tumor implantation, 40 days after tumor implantation and at the end of the anti-CXCR4 administration. 
These treatments had no significant effect on mouse body weight and the animals appeared healthy and active throughout the study. Tumor response was evaluated at the moment of sacrifice by determining tumor volume, calculated as (length)(width $2 / 2)$. We counted and measured macroscopic dissemination nodules throughout the entire peritoneal cavity and diaphragm. Also, we acquired complete liver, omentum, mesenteric tissue and lungs to check microscopic dissemination.

\section{Immunohistochemistry in paraffin-embedded samples and scoring}

Paraffin-embedded sections were deparaffinized in xylene and rehydrated in downgraded alcohols and distilled water. Antigens were retrieved under high-pressure conditions for 4 min in citrate buffer, $\mathrm{pH}$ 6, and endogen peroxidases were deactivated. Samples were blocked with goat normal serum before primary antibody incubation overnight at $4^{\circ} \mathrm{C}$. Rabbit polyclonal antiCXCR4 (Sigma-Aldrich), mouse monoclonal anti-Ki67 (NeoMarkers, Biocompare, Fremont, CA), rabbit polyclonal anti-GLUT-1 (Abcam, Cambridge, UK), rat monoclonal anti-mouse CD31 (Abcam) and rabbit polyclonal anti-Ecadherin (Cell Signaling Technologies, Beverly, MA) were used. Sections were incubated with the specific secondary anti-rabbit or anti-mouse EnVision antibodies (Dako, Agilent Technologies, Santa Clara, CA) or anti-rat biotinylated (Dako) before Streptavidin Horseradish Peroxidase Conjugate (Life Technologies, ThermoFisher Scientific) incubation, followed by the DAB developing system (Dako). Samples were counterstained with hematoxylin and visualized under light microscopy. 
We evaluated Ki67 only in the nucleus as the percentage of positivestaining tumor cells among total tumor cells. CXCR4, GLUT-1 and E-cadherin were measured as a grading scale, defined as follows: no detectable signal (0 points), low-intensity signal (1 point), moderate-intensity signal (2 points), or high-intensity signal (3 points). Labeling frequency was scored as the percentage of positive tumor cells. The multiplicative index of intensity and labeling frequency was used in our analysis, as previously described $(12,14)$. Mouse endothelial cells with CD31-positive staining were used to evaluate the microvascular density (MVD). We counted the number of CD31-positive vascular structures in five 400X hotspot fields on each tissue slide. The mean frequency of vessels per field in each sample was MVD.

Immunohistochemistry of CXCR4 and E-cadherin was performed on consecutive paraffin samples of OVA17 control tumors. We used a grid under the microscope to analysed how was the E-cadherin staining at positive CXCR4 zones. We counted the number of cells in each area and we calculated the respective percentatges.

\section{TUNEL assay}

We used an Apoptosis Detection Kit from Millipore (Billerica, MA) with paraffin-embedded tissue following the manufacturer's protocol exactly. We analyzed the central section of the tumors, evaluating apoptotic cells as the percentage of TUNEL positive-staining tumor cells among all tumor cells. 


\section{Immunofluorescence}

3- $\mu$ m-thick OCT tumor tissue sections were cut and used for double immunofluorescence staining. Mouse monoclonal anti-CD326 (EpCAM) conjugated to FITC (Miltenyi Biotec, Cologne, Germany) used as an epithelial tumoral cell marker and rabbit monoclonal anti-CXCR4 antibody (clone UMB2, Abcam) were simultaneously incubated overnight at $4^{\circ} \mathrm{C}$. Secondary goat antirabbit Alexa 546 or anti-mouse Alexa 488 was incubated for $1 \mathrm{~h}$. After washing, samples were mounted in VECTASHIELD with DAPI (Vector Laboratories, Burlingame, CA).

\section{Western blotting}

Samples from tumors were mechanically disrupted using RIPA lysis buffer and Western blot was performed as described elsewhere (12). Blots were incubated with 1/5000 mouse monoclonal anti-phospho-ERK1/2 (Thr202/Tyr204) antibody (Sigma-Aldrich), 1/3000 rabbit polyclonal anti-ERK1/2 total antibody (15), 1/1000 rabbit polyclonal anti-phospho-AKT (Ser 473) antibody (Cell Signaling Technologies), 1/1000 rabbit polyclonal anti-AKT antibody (Cell Signaling Technologies), 1/1000 rabbit polyclonal anti-phosphoSrc (Y418) antibody (Cell Signaling Technologies), 1/1000 rabbit polyclonal anti-Src antibody (Cell Signaling Technologies), 1/1000 rabbit polyclonal anti-ECadherin (24E10) antibody (Cell Signaling Technologies), 1/1000 rabbit polyclonal anti-PARP antibody (Cell Signaling Technologies), 1/5000 monoclonal mouse anti- $\alpha$-tubulin antibody (Sigma-Aldrich), or 1/5000 
monoclonal mouse anti-vinculin antibody (Sigma-Aldrich) in TBS containing 1\% non-fat dry milk overnight at $4^{\circ} \mathrm{C}$. Volumetric analysis was carried out using the Quantity One volume analysis tool (Bio-Rad, Hercules, CA).

\section{Quantitative real-time PCR}

Total RNA from tumors was extracted using the RNA Kit (Ambion, ThermoFisher Scientific). Total RNA from sorted cells was extracted using TRIZOL (Ambion). cDNA obtention and Real-time PCR of cDNA obtained was done as described elsewhere $(14,16)$. Specific human or mouse primers were designed for: human CXCR4 (5' TTTCCGTGAAGAAAATGCTA 3' and 5' TGTCCGTCATGCTTCTCAGT $\left.\quad 3^{\prime}\right) ; \quad$ mouse $\quad$ Cxcr4 CTTCCGGGATGAAAACGTCC 3' and 5' TGTCCGTCATGCTCCTTAGC 3'); human ZEB1 (5' TTTGGCTGGATCACTTTCAAG 3 ' and GCCAATAAGCAAACGATTCTG $\left.\quad 3^{\prime}\right) ; \quad$ human $\quad$ ZEB2 TGTAGATGGTCCAGAAGAAATG 3' and 5' CCATTGTTAATTGCGGTCT 3'); human SNAI1 (5' GCTGCAGGACTCTAATCCAGA 3 ' and 5' GACAGAGTCCCAGATGAGCAT 3') and human CDH-1 (E-cadherin) (5' CAGAATTGCTCACATTTCC 3' and 5' GGATTTGATCTGAACCAGGT 3'). Realtime PCR of cDNA obtained from tumors was carried out in a LightCycler instrument (Roche Molecular Biochemicals, Lewes, UK). The $\Delta \mathrm{C}_{t}$ values were calculated by subtracting the mean $C_{t}$ values of the $\beta$-actin gene from the mean $C_{t}$ values of each of the genes analyzed.

Human CXCR7 mRNA was quantified with a TAQMAN assay. 
Patient blood samples Real-Time PCR: Real Time PCR analysis was performed in a set of 20 healthy controls and 38 patients. RNA was purified with the QIAmp viral RNA mini kit (Qiagen, Hilden, Germany), specifically designed for very low cellularity samples. cDNA was synthesized by using SuperScriptIII chemistry (Invitrogen, Life Technologies, MA) following manufacturer's instructions. To further optimize the sensibility of detection, we performed a preamplification step by using the TaqMan PreAmp Master Mix kit (Applied Biosystems) with 14 reaction cycles. Preamplified products were subjected to TaqMan real-time PCR amplification. TaqMan probes Hs00607978_s1 (CXCR4), Hs00761767 s1 (CD19) and Hs00894734_m1 (CD45/PTPRC) were used.

CXCR4 expression values were analyzed using StepOne Software v.2.1 (Applied Biosystems, Life Technologies, CA), normalized to CD45 and represented as $(40-\Delta \mathrm{Ct})$, whereby $\Delta \mathrm{Ct}=$ duplicate mean (CtTARGET CtCD45).

\section{Cell viability assay}

Cell viability was determined as described elsewhere (12).

\section{Tissue and blood flow cytometry analyses}

CXCR4-positive and CXCR4-negative cells were obtained from SKOV-3GFP-Luciferase orthotopic ovarian tumors: When tumors attained a volume of 
$1500 \mathrm{~mm}^{3}$, animals were sacrificed, the tumors collected and mechanically and enzymatically disaggregated. Enzymatic digestion was performed by incubation with collagenase IV (2 U/ml; Gibco, ThermoFisher Scientific) plus Dispase II (4 $\mathrm{U} / \mathrm{ml}$, Gibco, ThermoFisher Scientific) for $10 \mathrm{~min}$ at $37^{\circ} \mathrm{C}$ with stirring. The cell suspension was passed through a $70-\mu \mathrm{m}$ filter and washed with erythrocyte lysis buffer (Ack Lonza, Basel, Switzerland). Isolated cells were stained with APC-labeled anti-human CD184 (CXCR4) mouse antibody (mouse IgG2a K, Clone 12G5 BioLegend, San Diego, CA) and DAPI. In all the studies, a minimum of 100,000 cells were analyzed. Cells were sorted using a Beckman Coulter MOFLO ASTRIOS flow cytometer system. GFP+CXCR+ and GFP + CXCR- cells were isolated.

Mouse blood was obtained from the saphenous vein, kept in an EDTA tube, diluted to $1 / 3$ with PBS $1 \mathrm{x}$ with $0.1 \% \mathrm{BSA}$ and $1.5 \mathrm{mM}$ EDTA before incubation with primary antibodies. Dead cells were eliminated with Hoechst 58 viability dye, the live cell population being between 100,000 and 300,000 cells. To identify circulating tumor cells, samples were stained with anti-human CD326 (EpCAM) mouse antibody (mouse lgG1k,. Clone HEA-125, Miltenyi Biotec) and APC-labeled anti-human CD184 (CXCR4) mouse antibody (mouse IgG2a K, Clone 12G5 BioLegend, San Diego, CA), incubated for 15 min and then washed with PBS $+0.1 \%$ BSA + 1.5 mM EDTA. Data were acquired with a Gallious $^{\mathrm{TM}}$ flow cytometer (Beckman Coulter, Brea, CA) and analyzed using the FCS Express 4 Image Cytometry program (De Novo Software, Los Angeles, $\mathrm{CA})$. 


\section{Statistical analyses}

Statistical analyses were done with SPSS for Windows 13.0 (SPSS, Inc., Chicago, IL). Differences between experimental and control groups were determined using the Mann-Whitney $U$ test.

\section{RESULTS}

As a first step towards evaluating the importance of CXCR4 in high-grade serous ovarian and endometrioid carcinomas, we performed immunohistochemical analysis to compare its expression in different primary tumors obtained from patients. As may be seen in Fig. 1A and B, tumors expressed different levels of CXCR4, with twice the level of expression in highgrade serous carcinomas than in endometrioid carcinomas (median CXCR4positive cells: $40 \%$ vs. $19.2 \%, p=0.0023$; median CXCR4 index: 80 vs. 40 ). This effect was independent of the FIGO stage: analysis of only the FIGO III stages of the two tumor subtypes yielded a near-identical result (median CXCR4positive cells: $40 \%$ for serous stage III vs. $9 \%$ for endometrioid stage III tumors, $\mathrm{p}=0.0018$; median CXCR4 index: 80 vs. 27) (Fig. 1C). Next we analyzed CXCR4 mRNA expression by RT-PCR between the two groups, confirming the results obtained by immunohistochemistry: endometrioid tumors had lower CXCR4 mRNA expression levels than did high grade serous tumors (Fig. 1D).

Given the previous results, we decided to study the relevance of CXCR4 expression to ovarian cancer progression in both tumor subtypes by using orthotopic preclinical models. To this end we used orthotopic models generated 
after implantation in the ovary of nude mice of tumor samples obtained from patients after surgery (PDX). We chose three ovarian orthotopic models previously generated by our group (12): two high-grade serous ovarian carcinoma that expressed high levels of CXCR4 (OVA17: evaluated by immunohistochemistry median CXCR4-positive cells $71 \%$, median \% of tumors with level 3 CXCR4 staining 16\%, Fig. 1E-G; and by RT-PCR, Fig. 1H) or intermediate levels (OVA8: evaluated by immunohistochemistry median CXCR4-positive cells $68 \%$, median \% of tumors with level 3 CXCR4 staining $6 \%$, Fig. 1E-G; and by RT-PCR, Fig. $1 \mathrm{H}$ ) and an endometrioid ovarian carcinoma model (OVA15) with low levels of CXCR4 expression (median CXCR4-positive cells $8 \%$, median \% of tumors with level 3 CXCR4 staining: $0 \%$, Fig. 1E-G; and by RT-PCR, Fig. $1 \mathrm{H})$. We confirmed these results by immunofluorescence (Suppl. Fig. 1A). CXCR4 could be expressed not only by tumoral cells but also by stromal cells present in the tumor. In order to discriminate CXCR4 expression in stromal (murine) and tumoral (human) cells, we designed specific mouse primers to measure mouse Cxcr4 mRNA. In contrast with the results obtained for human CXCR4, we did not detect any differences between the high-grade serous tumors and the endometrioid carcinomas in stromal Cxcr4 mRNA (Suppl. Fig. 1B). Finally, we also measured mRNA levels of CXCR7, a receptor of the same family as CXCR4, which also binds CXCL12. This receptor was expressed at very low levels in tumoral cells from OVA17 and OVA15 tumors, while OVA8 tumors presented similar CXCR7 mRNA levels relative to those of HeLa cells (Suppl. Fig. 1C). 
To evaluate the importance of CXCR4 we first used AMD3100, a small molecule antagonist of CXCR4 $(17,18)$, and studied its effect on OVA17, OVA8 and OVA15 tumor growth. Mice bearing palpable tumors were randomized into two groups and treated with vehicle or AMD3100 for 4 weeks. In the case of OVA17 and OVA8 the final tumor volume of the treated mice was smaller $(60 \%$ and $48 \%$ less, respectively) (Fig. 2A and B). In contrast, the effects of AMD3100 in the endometrioid OVA15 model were clearly weaker than in the serous models, with AMD3100 causing a 30\% reduction in tumor volume (Fig. 2C). As expected, treated tumors had fewer tumoral CXCR4-positive cells as measured by RT-PCR (Suppl. Fig. 2A-C).

These results prompted us to determine how AMD3100 reduced tumor volume in our orthotopic models. H\&E staining showed no significant differences in tumor fibrosis or necrosis in OVA17, OVA8 and OVA15 tumors treated with AMD3100 compared with controls (Suppl. Fig. 3A-C). We also did not note any effect on Ki67 staining levels, indicating a lack of effect of CXCR4inhibition on cell proliferation (Suppl. Fig. 3D and E). Given these results, we next examined the putative effect of AMD3100 on cell apoptosis by TUNEL staining of tumoral sections. Administration of AMD3100 induced an increase in apoptotic cells in AMD-treated tumors compared with untreated tumors in all the models analyzed (Fig. 2D), although the effect was more pronounced in OVA17 (88\% increase) and in OVA8 (105\% increase) than in OVA15 (28\% increase). These results were confirmed by immunodetection of the cleaved form of PARP in controls or AMD3100-treated OVA17 tumors (Fig. 2E and F). We also analyzed the effect of AMD3100 treatment on angiogenesis. AMD3100 induced 
a significant reduction in the number of vessels in OVA17 (30\%) and OVA15 tumors (33\%) (Suppl. Fig. 4A and B). These results were confirmed by immunohistochemistry by measuring the increase in a hypoxia-response protein, the glucose transporter GLUT1. AMD3100-treatment caused a clear and significant increase in GLUT1 expression in OVA17 (x3.3) and OVA15 (x5) tumors compared with controls (Suppl. Fig. 4C and D). Overall, these results imply that this anti-angiogenic effect could contribute to the observed outcomes, but combined with a direct anti-tumoral cell effect on tumors expressing high levels of CXCR4, as OVA17.

Consistent with their more aggressive and metastatic potential, OVA17 and OVA8 serous tumors presented macroscopic tumor peritoneal dissemination (Fig. 3A and B). By contrast, the OVA15 endometrioid model did not show any macroscopic or microscopic dissemination. These tumor nodules were greatly reduced in number and size in the AMD3100-treated groups (Fig. $3 \mathrm{~A}$ and $\mathrm{B})$. We then investigated the presence of CTCs in the blood of tumorimplanted mice, using flow cytometry to detect human EPCAM-positive cells. We compared the number of CTCs in the blood of animals 40 days after tumor implantation with that in blood of control animals without tumors (Sham, Fig. 3C). These CTCs were present until the day they were sacrificed (2 months after implantation). CTC levels were high in OVA17, intermediate in OVA8 and not present in OVA15. Moreover, $60 \%$ of the circulating CTC EPCAM+ cells were also positive for CXCR4 (Suppl. Fig. 5). When animals were treated with AMD3100, all CTCs present in OVA17 and OVA8-implanted animals completely disappeared (Fig. 3C), in parallel with the disappearance of tumoral 
dissemination. In order to confirm these results, we analyzed CXCR4 mRNA expression levels in blood cells from controls or ovarian carcinoma affected patients. As may be seen in Fig. 3D, blood cells expressed twice the level of CXCR4 expression in carcinoma affected patients than in blood from controls. To confirm the epithelial phenotype of the blood cells analyzed we also measured cytokeratin19 mRNA levels, a well known epithelial marker. As shown in Suppl. Fig. 6, cytokeratine19 was also increased in blood from patients compared to controls. Besides, we observed a strong correlation between the expression levels of CXCR4 and cytokeratin19 $\left(r^{2}=0,83\right.$ and $p<0.0001$, according to Pearson test), reinforcing the tumoral and epithelial composition of our blood patient enriched fraction.

Next we specifically inhibited CXCR4 expression in SKOV-3 cells, a widely used cell model for peritoneal invasion studies $(19,20)$. These cells did not expressed CXCR4 when cultured in vitro (Fig. 4A), but when cells were injected into the ovary of nude mice and tumors were generated, human CXCR4 was up-regulated at the mRNA and protein levels (Fig. 4A-B and Suppl. Fig. 7). SKOV-3-shNS and SKOV-3-shCXCR4 cells were generated by transducing lentiviral vectors expressing either CXCR4-shRNAs or a negative control using a non-silencing vector. We used two independent shRNA CXCR4 vectors (sh865 and sh863) that reduced CXCR4 mRNA and protein expression in SKOV-3-shCXCR4 tumors by $60-80 \%$ (Fig. 4A-B). As expected, shCXCR4 expression in cultured SKOV-3 cells did not significantly affect colony formation capacity or cell viability (Suppl. Fig. 8A and B). In contrast, in vivo tumors generated from SKOV-3-shCXCR4 cells grew more slowly than those from 
SKOV-3-shNS cells (Fig. 4C), implying a role for CXCR4 in the ability of cells to proliferate. Microvascular density in these SKOV-3-shCXCR4-derived tumors was also affected, with a decrease of $40 \%$ in the number of vessels (Suppl. Fig. $8 C)$. When we compared the capacity of similarly sized tumors to disseminate, SKOV-3-shCXCR4 tumors presented no peritoneal dissemination, unlike SKOV-3-shNS tumors (Fig. 4D and E). Moreover, according to this blocking of disseminative capacity, CTCs were present at high levels in SKOV-3-shNS inoculated animals but at very low levels in SKOV-3-shCXCR4 (Fig. 4F and Suppl. Fig. 9), confirming the role of CXCR4 in ovarian tumoral cell blood mobilization.

We then analyzed the various signaling pathways already known to be affected by CXCR4 stimulation. Activated Src levels (Src phosphorylated on tyrosine 418 ) in OVA17 tumoral samples decreased by $50 \%$ following CXCR4 inhibition with AMD3100 (Fig. 5A and B). We did not detect any change in AKT phosphorylation in tumors treated with AMD3100 compared with controls (Fig. 5A), while activated ERK1/2 also decreased by $66 \%$ (Fig. 5A and C). Similar results were obtained when we analyzed active ERKs, active AKT and active Src in SKOV-3-shNS and SKOV-3-shCXCR4 tumors (Suppl. Fig. 10A-D). In contrast, no significant change in active Src and active ERKs was observed when we treated OVA15 tumors with AMD3100 (Suppl. Fig. 10E-G).

Fang et al. recently reported that ERKs and Src activation stimulated EMT in ovarian cancer cells (21). These results prompted us to study the effect of CXCR4 inhibition on some proteins involved in EMT. First, we isolated CXCR4-positive cells from SKOV-3-GFP ovarian orthotopic tumors using flow 
cytometry. Cells were selected, first depending on GFP expression to provide us only of human tumoral cells rather than mice stromal cells (Suppl. Fig. 11). After, CXCR4 was detected in the GFP positive population and we only sorted the most positive and the most negative CXCR4 populations. In these cells we compared the mRNA expression levels of ZEB1, ZEB2, SNAl1 and CDH-1 (Ecadherin) with those in CXCR4-negative tumoral cells. CXCR4-positive cells expressed low levels of $\mathrm{CDH}-1$ (an epithelial marker) and high levels of the mesenchymal markers ZEB1, ZEB2 and SNAI1 compared with CXCR4negative cells (Fig. 5D). We confirmed these results by measuring E-cadherin by western blot in tumors generated from SKOV-3-shNS or shCXCR4 cells: we found low levels of E-cadherin in control SKOV-3-shNS tumors, which increased when CXCR4 levels decreased by blocking CXCR4 expression (Fig. 5E and F). Moreover, E-cadherin levels were also low in OVA17 control tumors, but increased after AMD3100 treatment (Fig. 5G and H). Finally, we analyzed the presence of CXCR4 and E-cadherin in OVA17 control tumors samples by immunohistochemistry. As shown in Suppl. Fig. 12 and Fig.5I, 78\% of cells positive for CXCR4 were negative for E-cadherin and only $22 \%$ of cells positive for CXCR4 were also positive for E-cadherin. In contrast, $55 \%$ of cells negative for CXCR4 were positive for E-cadherin, confirming that CXCR4 expression and its signaling induced a more mesenchymal phenotype.

\section{DISCUSSION}

Our work highlights CXCR4 as being a key player in ovarian serous cancer progression, eliciting mobilization of tumoral CXCR4-positive cells and 
an increase in its capacity to metastasize. Our study also identifies a dual role for CXCR4 in primary tumor progression. First, in some tumoral models CXCR4, expressed by tumoral or stromal cells (as myeloid or T cells), collaborates with angiogenic factors to generate new vessels. CXCL12 and VEGF-A synergize to stimulate proliferation and migration of HUVEC, inducing in vivo angiogenesis in mouse models (10). Also, hypoxia stimulates production of CXCL12 and VEGF-A in ovarian tumoral cells, and cell-derived ovarian tumors treated with AMD3100 or with an anti-CXCL12 RNAi had lower vessel densities than controls (22). In addition to this anti-angiogenic effect, our results indicate that CXCR4 plays a direct role in tumoral progression. It has been reported that CXCR4 siRNA directly affects cell viability and blocks the cell cycle in G1/S in ovarian A2780 cells (9), and cell proliferation in IGROV, CAOV3 and primary ovarian tumor cells isolated from ascites (23). In vivo, AMD3100 treatment of animals with intraperitoneally injected BR5-1 ovarian cancer cells brought about a reduction in tumor weight (22). Our results in tumors overexpressing shRNAs against CXCR4 confirm these results. They also indicate a stronger effect of AMD3100, reducing tumoral growth and inducing apoptosis in orthotopic tumors with higher CXCR4 levels than in those with lower levels. Taken together, these observations indicate that anti-CXCR4 treatments influence tumoral growth by a combination of effects comprising indirect anti-angiogenic and direct anti-tumoral activities in ovarian CXCR4positive tumoral cells.

The CXCR4-CXCL12 axis participates in metastatic spreading in various tumors, such as those of the breast and prostate $(4,5)$. There have been 
several reports of a correlation between CXCR4 expression and the stage of ovarian tumors (low in FIGO stages I and II, high in stages III and IV) and with the presence of metastasis in ovarian cancer patients (6-9). Moreover, in vivo intraperitoneal injection of ES-2 cells caused the formation of peritoneal tumors that disseminated throughout the entire cavity, while AMD3100 suppressed this effect (7). Our work with orthotopic ovarian cancer models confirmed this CXCR4 role in peritoneal dissemination and that AMD3100 treatment completely blocks this process. Moreover, our analysis of human samples from patients indicate a higher level of CXCR4 expression in high-grade serous carcinomas than in endometrioid carcinomas, a correlation that also reinforces the role of this cytokine receptor in enabling ovarian cancer to disseminate and metastasize. However, more importantly, our results identify for the first time a role for CXCR4 in inducing and maintaining CTCs present in blood. Circulating tumoral cells have received a great deal of attention from researchers in recent years because of their predictive and prognostic value as biomarkers (24). CTCs have been detected in ovarian cancer patients $(25,26)$, and their concentration in blood predicts progression-free and overall survival in advanced ovarian cancer patients (27). Our preclinical models showed a strong correlation between the level of CTCs in blood and peritoneal dissemination capacity. It has been shown in ovarian tumors that peritoneal dissemination to the omentum can occur via hematogenous metastasis and the presence of CTCs (20). These cells had high levels of ErbB3, and in consequence, high levels of Src and Rac-1 activity and greater migratory capacity. Fang et al. have also reported that ERKs and Src activation stimulated EMT in ovarian cancer cells, increasing their migratory capacities (21). Our results lead us to propose 
an alternative signaling pathway, that of CXCR4 activation, by which Src/ERKs, induction of EMT and the capacity to migrate and generate CTCs are stimulated. As a consequence, we propose that CXCR4 and Src inhibitors may be therapeutic agents for the alternative or complementary treatment of advanced epithelial serous ovarian cancer patients.

\section{ACKNOWLEDGEMENTS}

We are grateful to the subjects who participated in this study.

\section{REFERENCES}

1. Bast RC, Jr., Hennessy B, Mills GB. The biology of ovarian cancer: new opportunities for translation. Nat Rev Cancer 2009 Jun;9(6):415-28.

2. Bowtell DD. The genesis and evolution of high-grade serous ovarian cancer. Nat Rev Cancer 2010 Nov;10(11):803-8.

3. Vaughan S, Coward JI, Bast RC, Jr., et al. Rethinking ovarian cancer: recommendations for improving outcomes. Nat Rev Cancer 2011 Oct;11(10):719-25.

4. Weitzenfeld P, Ben-Baruch A. The chemokine system, and its CCR5 and CXCR4 receptors, as potential targets for personalized therapy in cancer. Cancer Lett 2014 Sep 28;352(1):36-53.

5. Domanska UM, Kruizinga RC, Nagengast WB, et al. A review on CXCR4/CXCL12 axis in oncology: no place to hide. Eur J Cancer 2013 Jan;49(1):219-30. 
6. Jiang $Y P, W u X H$, Shi $B, W u$ WX, Yin GR. Expression of chemokine CXCL12 and its receptor CXCR4 in human epithelial ovarian cancer: an independent prognostic factor for tumor progression. Gynecol Oncol 2006 Oct;103(1):226-33.

7. Kajiyama H, Shibata K, Terauchi M, Ino K, Nawa A, Kikkawa $F$. Involvement of SDF-1alpha/CXCR4 axis in the enhanced peritoneal metastasis of epithelial ovarian carcinoma. Int J Cancer 2008 Jan 1;122(1):91-9.

8. Barbolina MV, Kim M, Liu Y, et al. Microenvironmental regulation of chemokine (C-X-C-motif) receptor 4 in ovarian carcinoma. Mol Cancer Res 2010 May;8(5):653-64.

9. Li J, Jiang K, Qiu X, et al. Overexpression of CXCR4 is significantly associated with cisplatin-based chemotherapy resistance and can be a prognostic factor in epithelial ovarian cancer. BMB Rep 2014 Jan;47(1):33-8.

10. Kryczek I, Lange A, Mottram P, et al. CXCL12 and vascular endothelial growth factor synergistically induce neoangiogenesis in human ovarian cancers. Cancer Res 2005 Jan 15;65(2):465-72.

11. Labrosse B, Brelot A, Heveker N, et al. Determinants for sensitivity of human immunodeficiency virus coreceptor CXCR4 to the bicyclam AMD3100. Journal of Virology 1998;72(8):6381-8.

12. Alsina-Sanchis E, Figueras A, Lahiguera A, et al. The TGFbeta pathway stimulates ovarian cancer cell proliferation by increasing IGF1R levels. Int $J$ Cancer 2016 Oct 15;139(8):1894-903.

13. Ibrahimi A, Vande Velde G, Reumers V, et al. Highly efficient multicistronic lentiviral vectors with peptide 2A sequences. Human Gene Therapy 2009;20:845-60. 
14. Juliachs M, Munoz C, Moutinho CA, et al. The PDGFRbeta-AKT pathway contributes to CDDP-acquired resistance in testicular germ cell tumors. Clin Cancer Res 2014 Feb 1;20(3):658-67.

15. McKenzie FR, Pouyssegur J. CAMP-mediated growth inhibition in fibroblasts is not mediated via mitogen-activated protein (MAP) kinase (ERK) inhibition. cAMP-dependent protein kinase induces a temporal shift in growth factor-stimulated MAP kinases. J Biol Chem 1996 Jun 7;271(23):13476-83.

16. Castillo-Avila W, Piulats JM, Garcia Del Muro X, et al. Sunitinib inhibits tumor growth and synergizes with cisplatin in orthotopic models of cisplatinsensitive and cisplatin-resistant human testicular germ cell tumors. Clin Cancer Res 2009 May 15;15(10):3384-95.

17. Donzella GA, Schols D, Lin SW, et al. AMD3100, a small molecule inhibitor of HIV-1 entry via the CXCR4 co-receptor. Nat Med 1998 Jan;4(1):727.

18. Hatse S, Princen K, Bridger G, De Clercq E, Schols D. Chemokine receptor inhibition by AMD3100 is strictly confined to CXCR4. FEBS Lett 2002 Sep 11;527(1-3):255-62.

19. Nieman KM, Kenny HA, Penicka CV, et al. Adipocytes promote ovarian cancer metastasis and provide energy for rapid tumor growth. Nature Medicine 2011;17(11):1498-503.

20. Pradeep S, Kim SW, Wu SY, et al. Hematogenous metastasis of ovarian cancer: rethinking mode of spread. Cancer Cell 2014 Jul 14;26(1):77-91.

21. Fang $\mathrm{D}$, Chen $\mathrm{H}$, Zhu JY, et al. Epithelial-mesenchymal transition of ovarian cancer cells is sustained by Rac1 through simultaneous activation of 
MEK1/2 and Src signaling pathways. Oncogene 2016;doi: 10.1038/onc.2016.323.

22. Righi E, Kashiwagi S, Yuan J, et al. CXCL12/CXCR4 blockade induces multimodal antitumor effects that prolong survival in an immunocompetent mouse model of ovarian cancer. Cancer Res 2011 Aug 15;71(16):5522-34.

23. Scotton CJ, Wilson JL, Scott K, et al. Multiple actions of the chemokine CXCL12 on epithelial tumor cells in human ovarian cancer. Cancer Res 2002 Oct 15;62(20):5930-8.

24. Alix-Panabieres C, Pantel K. Challenges in circulating tumour cell research. Nat Rev Cancer 2014 Sep;14(9):623-31.

25. Pecot CV, Bischoff FZ, Mayer JA, et al. A novel platform for detection of CK+ and CK- CTCs. Cancer Discov 2011 Dec;1(7):580-6.

26. Phillips KG, Velasco CR, Li J, et al. Optical quantification of cellular mass, volume, and density of circulating tumor cells identified in an ovarian cancer patient. Front Oncol 2012;2:72.

27. Poveda A, Kaye SB, McCormack R, et al. Circulating tumor cells predict progression free survival and overall survival in patients with relapsed/recurrent advanced ovarian cancer. Gynecol Oncol 2011 Sep;122(3):567-72. 


\section{FIGURE LEGENDS}

Figure 1. The level of CXCR4 expression is higher in high-grade serous than in endometrioid human ovarian carcinomas.

A) High-grade serous and endometrioid ovarian carcinomas sections were immunostained for CXCR4. Pictures representing different intensity levels of tumor-positive cells are shown. 400X, scale bars, $50 \mu \mathrm{m}$.

B) CXCR4 index (using the multiplicative index of the intensity of the stain and the labeling frequency) by immunohistochemistry assay from 31 high-grade serous tumors and 30 endometrioid tumors. ${ }^{* *}, p<0.001$ (two-tailed MannWhitney U test).

C) CXCR4 index by immunohistochemistry from 31 high-grade serous and 5 high-grade endometrioid tumors. *, $p<0.05$ (two-tailed Mann-Whitney U test).

D) mRNA levels of human CXCR4 analyzed by quantitative real-time PCR in normal human Falopian tube epithelium (6 samples), high grade serous (7 samples) and endometrioid (6 samples) ovarian tumors. Results are expressed as the mean percentage and SEM of mRNA expression levels.

E) Sections from OVA17 and OVA8 high-grade serous and OVA15 endometrioid ovarian tumors were immunostained for CXCR4. 200X (Scale bars, $100 \mu \mathrm{m}$ ) and 400X (Scale bars, $50 \mu \mathrm{m}$ ).

F) Quantification of CXCR4 levels (using the multiplicative index) in tumor tissue sections from OVA17 $(n=7)$, OVA8 $(n=5)$ or OVA15 $(n=4)$ tumor samples. Error 
bars indicate the standard errors of the mean (SEMs). ${ }^{*}, p<0.05 ;{ }^{* *} ; \mathrm{p}<0.01$ (two-tailed Mann-Whitney U test).

G) Quantification of more positive CXCR4 staining (\% of cells with staining level $3)$ in tumor tissue sections from OVA17 $(n=7)$, OVA8 $(n=5)$ and OVA15 $(n=4)$ tumor samples. Error bars indicate the standard errors of the mean (SEMs).

H) mRNA levels of human CXCR4 analyzed by quantitative real-time PCR in OVA17 (7 samples), OVA8 $(n=5)$ and OVA15 (5 samples) orthotopic ovarian tumors. Results are expressed as the mean percentage and SEM of mRNA expression levels relative to the OVA17 group. ${ }^{* *}, \mathrm{p}<0.01$ (two-tailed MannWhitney $\mathrm{U}$ test).

Figure 2. Blocking of CXCR4 activity inhibits tumor growth in xenograft orthotopic models of epithelial ovarian tumors by inducing apoptosis.

A) Mice with orthotopically implanted OVA17 ovarian tumors were treated with vehicle or AMD3100 for 4 weeks. Final volumes are illustrated by a boxplot. Results are the mean and SEM of 10 control tumors and 10 AMD3100-treated tumors. *, $p<0.05$ (two-tailed Mann-Whitney $U$ test).

B) Mice with orthotopically implanted OVA8 ovarian tumors were treated with vehicle or AMD3100 for 4 weeks. Final volumes are illustrated by a boxplot. Results are the mean and SEM of 5 control tumors and 5 AMD3100-treated tumors. $\mathrm{p}=0.095$ (two-tailed Mann-Whitney $\mathrm{U}$ test). 
C) Mice with orthotopically implanted OVA15 ovarian tumors were treated with vehicle or AMD3100 for 4 weeks. Final volumes are illustrated by a boxplot. Results for OVA15 are the mean and SEM of 5 control tumors and 5 AMD3100treated tumors. $\mathrm{p}=0.2$ (two-tailed Mann-Whitney $U$ test).

D) Sections from OVA17, OVA8 and OVA15 control and AMD3100-treated tumors were stained for TUNEL. Results are expressed as the percentage of TUNEL positive-staining nuclei relative to the control group, and are the mean and SEM of 7 control and 6 AMD3100-treated OVA17 tumors, of 4 control and 5 AMD3100-treated OVA8 tumors and of 4 control and 4 AMD3100-treated OVA15 tumors. *, $p<0.05$ (two-tailed Mann-Whitney U test).

E) Expression of PARP and vinculin were analyzed by western blot in AMD3100-treated and control OVA17 tumors. A representative blot showing results from 3 independent control tumors and 4 independent AMD3100-treated tumors.

F) Densitometric quantifications of cleaved PARP relative to total PARP are shown (\%) in AMD3100-treated and control OVA17 tumors. Results are the mean and SEM of 5 control tumors and 5 AMD3100-treated tumors. ${ }^{*}, p<0.05$ (two-tailed Mann-Whitney U test).

Figure 3. CXCR4 inhibition blocks hematogenous dissemination.

A) Number of intraperitoneal dissemination nodules/mouse. Results are the mean and SEM of 9 controls and 9 AMD-treated OVA17 tumors, 6 controls and 
5 AMD-treated OVA8 tumors and of 4 controls and 4 AMD-treated OVA15 tumors. ${ }^{* \star}, p<0.001$ (two-tailed Mann-Whitney U test).

B) Volume of peritoneal dissemination nodules. Numbers indicate the mean volume in $\mathrm{mm}^{3}$ of each group. Results are the mean and SEM of 9 controls and 9 AMD-treated OVA17 tumors, 6 controls and 5 AMD-treated OVA8 tumors and of 4 controls and 4 AMD-treated OVA15 tumors. ${ }^{* \star *}, p<0.001$ (two-tailed MannWhitney $\mathrm{U}$ test).

C) Number of CTCs (human EPCAM-positive cells) present in mouse blood from animals with the indicated tumors orthotopically implanted for 40 days. Results are expressed as the mean and SEM of the percentage of EPCAMpositive cells of all live cells in blood from 9 sham animals, 5 OVA17-implanted animals, 4 OVA17 AMD3100-treated animals, 6 OVA8-implanted animals and 5 OVA8 AMD3100-treated animals, and 4 OVA15-implanted animals and 4 OVA15 AMD3100-treated animals. $\mathrm{p}=0.063$ between OVA17 control and AMD3100-treated groups (two-tailed Mann-Whitney U test).

D) mRNA levels of human CXCR4 analyzed by quantitative real-time PCR in blood cells from controls $(n=20)$ or in patients with high grade serous ovarian tumors $(n=38)$. Results are illustrated by a boxplot and are expressed as the mean percentage and SEM of mRNA expression levels normalized by CD45 mRNA expression. ${ }^{* \star}, \mathrm{p}<0.001$ (two-tailed Mann-Whitney $\mathrm{U}$ test).

Figure 4. shRNA block of CXCR4 expression inhibits circulating tumoral cells in mouse blood and peritoneal dissemination. 
A) mRNA levels of human CXCR4 analyzed by quantitative real-time PCR in OVA17 $(n=7)$, SKOV-3 cells $(n=6)$, SKOV-3 parental orthotopic ovarian tumors (Par, $n=10$ ), SKOV-3-sh non-silencing control orthotopic ovarian tumors (shNS, $n=3)$, SKOV-3-shCXCR4 863 orthotopic ovarian tumors $(n=5)$ and SKOV-3shCXCR4 865 orthotopic ovarian tumors $(n=5)$. Results are expressed as the mean and SEM of mRNA expression levels. ${ }^{*}, p<0.05,{ }^{* * *}, p<0.001$ (two-tailed Mann-Whitney U test).

B) Quantification of CXCR4 levels (using the multiplicative index) in tumor tissue sections from SKOV-3 parental orthotopic ovarian tumors (Par, $n=3$ ), SKOV-3-sh control orthotopic ovarian tumors (shNS, n=3), SKOV-3-shCXCR4 863 orthotopic ovarian tumors $(n=8)$ and SKOV-3-shCXCR4 865 orthotopic ovarian tumors $(n=4)$. Error bars indicate the SEMs. ${ }^{* *}, p<0.01$ (two-tailed Mann-Whitney U test).

C) SKOV-3 parental, SKOV-3-shNS, SKOV-3-shCXCR4 863 and SKOV-3shCXCR4 865 cells were injected in the ovary of nude mice. After the times indicated, the tumor volume was measured by palpation. Results are the mean and SEM of 3 independent tumors for each clone.

D) Number of intraperitoneal dissemination nodules/mouse in animals with tumors generated from the indicated SKOV-3 clones at the moment of sacrifice. Results are expressed as the mean and SEM of 4 SKOV-3-sh control orthotopic ovarian tumors (shNS), 6 SKOV-3-shCXCR4 863 orthotopic ovarian tumors and 4 SKOV-3-shCXCR4 865 orthotopic ovarian tumors. ${ }^{*}, p<0.05 ;{ }^{* *}, p<0.01$ (twotailed Mann-Whitney U test). 
E) Volume of peritoneal dissemination nodules in animals with tumors generated from the indicated SKOV-3 clones at the moment of sacrifice. Numbers indicate the mean volume in $\mathrm{mm}^{3}$ in every group. Results are the mean and SEM of 4 SKOV-3-sh control orthotopic ovarian tumors (shNS), 6 SKOV-3-shCXCR4 863 orthotopic ovarian tumors and 4 SKOV-3-shCXCR4 865 orthotopic ovarian tumors. ${ }^{*}, \mathrm{p}<0.05 ;{ }^{* *}, \mathrm{p}<0.01$ (two-tailed Mann-Whitney $U$ test).

F) Number of CTCs (human EPCAM-positive cells) present in mouse blood from animals with tumors generated from the indicated SKOV-3 clones of the same volume. Results are expressed as the mean and SEM of the percentage of EPCAM-positive cells of all live cells in sham animals ( $n=9)$, SKOV-3-sh control orthotopic ovarian tumor-implanted animals (shNS, n=4), SKOV-3shCXCR4 863 orthotopic ovarian tumors implanted animals $(n=5)$ and SKOV-3shCXCR4 865 orthotopic ovarian tumors implanted animals $(n=5) .{ }^{*}, p<0.05$ (two-tailed Mann-Whitney U tests).

Figure 5. CXCR4 inhibition blocks Src and ERKs activation and epithelialmesenchymal transition.

A) Expression of phosphorylated $\mathrm{Src}$ ( $\mathrm{Tyr} 418, \mathrm{pSrc}$ ), total $\mathrm{Src}$ (T-Src), phosphorylated AKT (Ser 473, pAKT), total AKT (T-AKT, phosphorylated ERK1/2 (Thr202/Tyr204, pERK1/2), total ERK1/2 (T-ERK1/2) and tubulin was analyzed by western blot in AMD3100-treated and control OVA17 tumors. A 
representative blot showing results from 3 independent control tumors and 3 independent AMD3100-treated tumors is shown.

B) Densitometric quantification of pSrc normalized with respect to total Src. Results are the mean and SEM in arbitrary units of 5 control tumors and 6 AMD3100-treated OVA17 tumors. *, $p<0.05$ (two-tailed Mann-Whitney U tests).

C) Densitometric quantification of $\mathrm{pERK} 1 / 2$ normalized with respect to total ERK1/2. Results are the mean and SEM in arbitrary units of 5 control tumors and 8 AMD3100-treated OVA17 tumors. ${ }^{*}, \mathrm{p}<0.05$ (two-tailed Mann-Whitney U tests).

D) mRNA levels of human ZEB1, ZEB2, SNAl1 and CDH-1 (E-cadherin) were analyzed by quantitative real-time PCR in CXCR4-positive and CXCR4negative cells isolated from SKOV-3-GFP-Luciferase orthotopic ovarian tumors $(n=3)$. Results are expressed as the mean and SEM of mRNA expression levels in CXCR4-positive cells related to mRNA expression levels in CXCR4-negative cells.

E) Expression of E-cadherin and vinculin was analyzed by western blot in SKOV-3-sh control orthotopic ovarian tumors (shNS, $n=3$ independent tumors), SKOV-3-shCXCR4 863 orthotopic ovarian tumors ( $n=4$ independent tumors) and SKOV-3-shCXCR4 865 orthotopic ovarian tumors ( $n=3$ independent tumors). A representative blot is shown.

F) Densitometric quantification of E-cadherin normalized with respect to vinculin. Results are the mean and SEM in arbitrary units of SKOV-3-sh control 
(shNS, $n=4)$, SKOV-3-shCXCR4 $863(n=9)$ and SKOV-3-shCXCR4 $865(n=7)$ orthotopic ovarian tumors. ${ }^{*}, p<0.05$ (two-tailed Mann-Whitney U tests).

G) Expression of E-cadherin and vinculin was analyzed by western blot in control OVA17 tumors ( $\mathrm{n}=4$ independent tumors) and in AMD3100-treated OVA17 tumors ( $\mathrm{n}=4$ independent tumors). A representative blot is shown.

H) Densitometric quantification of E-cadherin normalized with respect to vinculin. Results are the mean and SEM in arbitrary units of 4 control tumors and 7 AMD3100-treated OVA17 tumors. ${ }^{*}, \mathrm{p}<0.05$ (two-tailed Mann-Whitney U tests).

I) Percentage of positive or negative E-cadherin tumoral cells that were also positive or negative for CXCR4 staining in consecutive paraffine sections from control OVA17 tumors ( $n=4$ independent tumors). 
Figure 1

A)

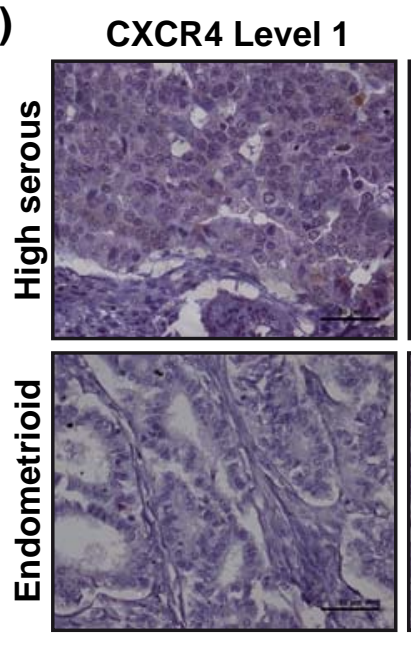

CXCR4 Level 2
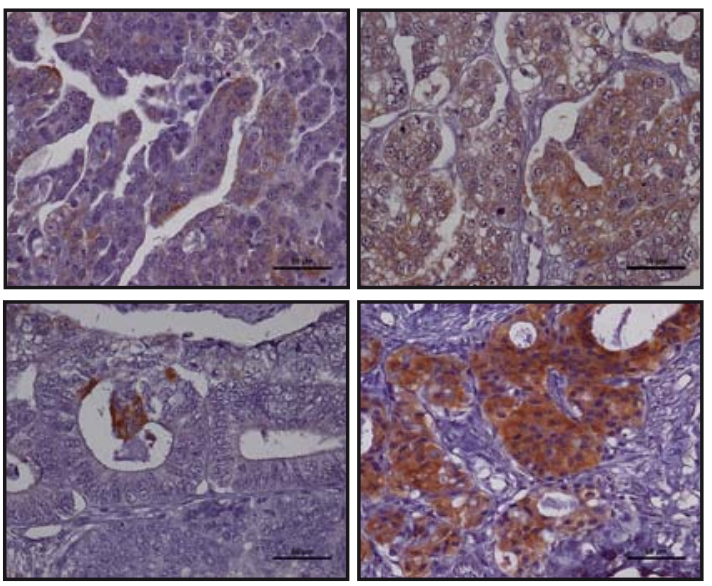

B)

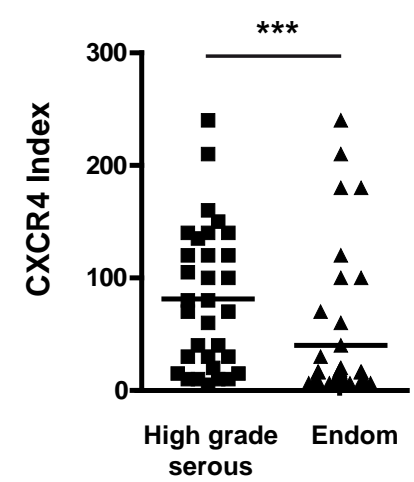

E)

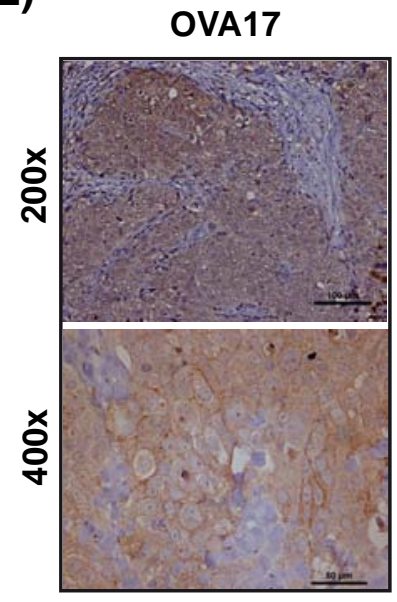

F)

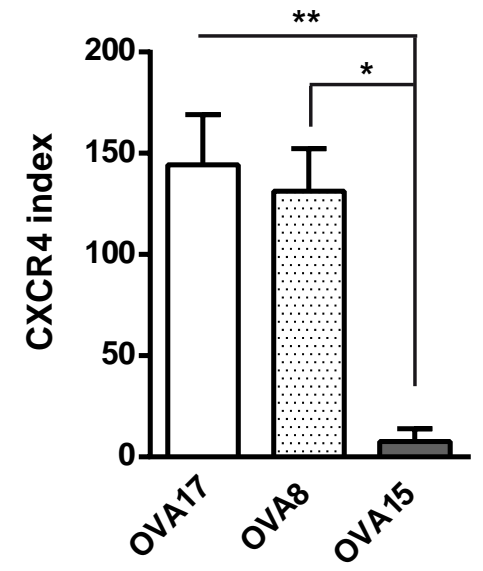

C)

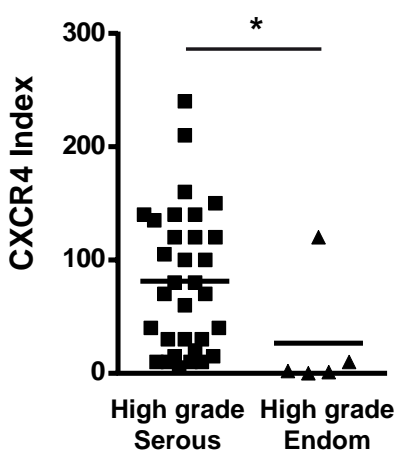

OVA8

OVA15

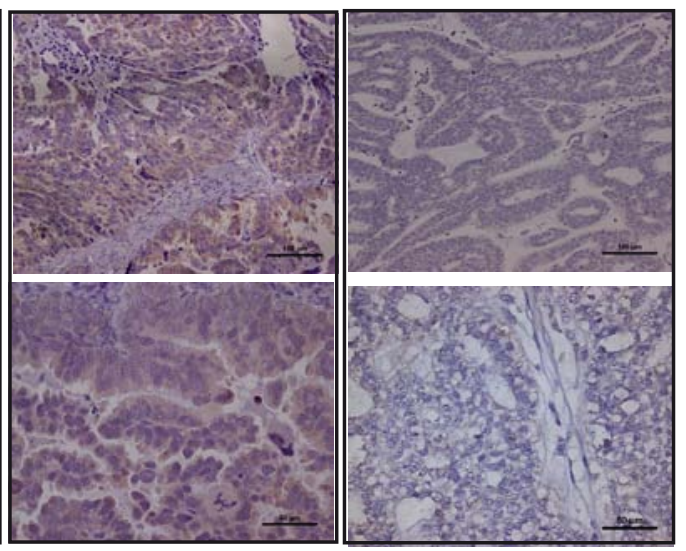

G)

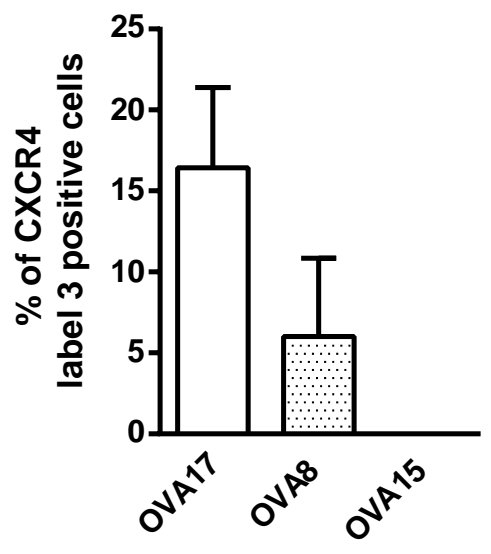

D) CXCR4 mRNA

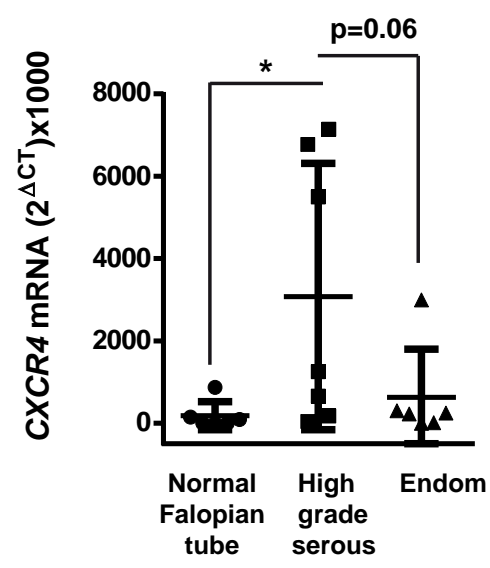

H)
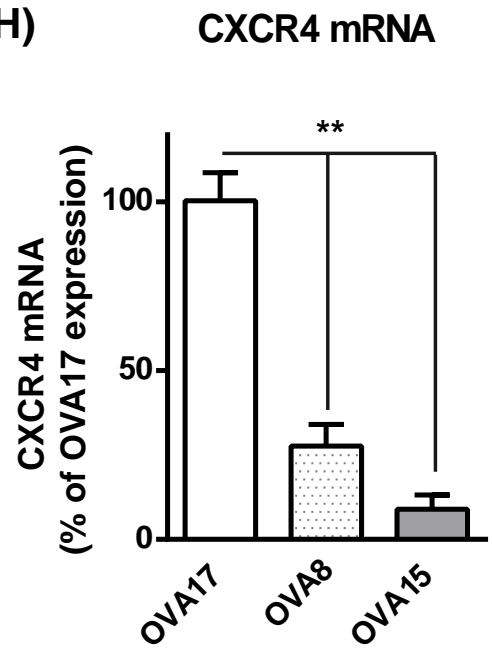
Figure 2

A)

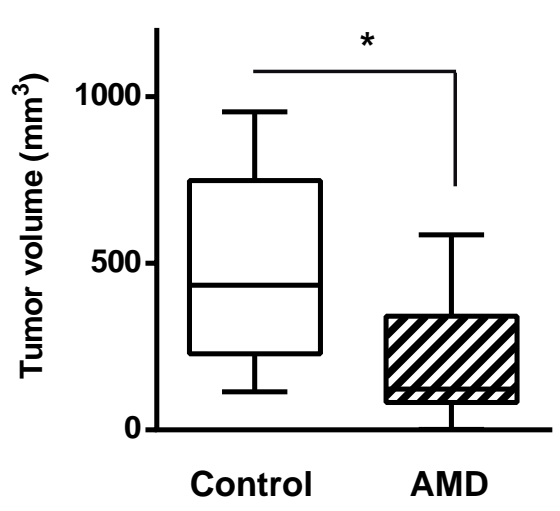

D)

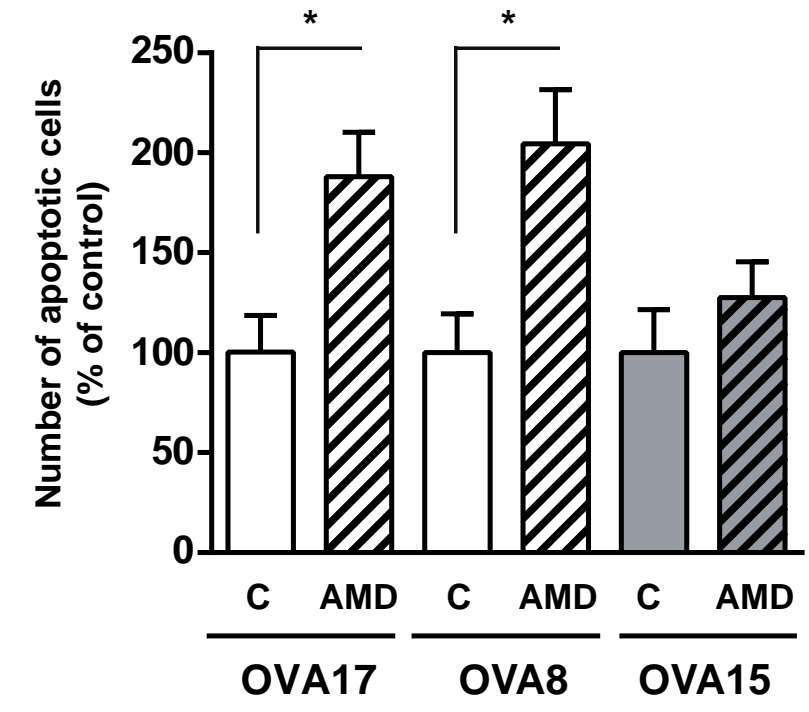

B)

OVA8

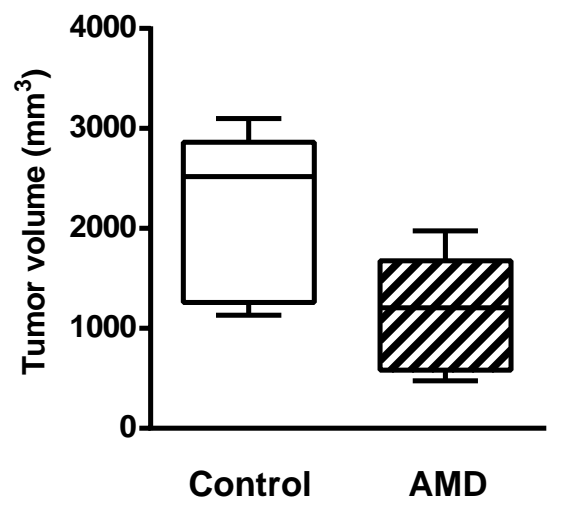

E)

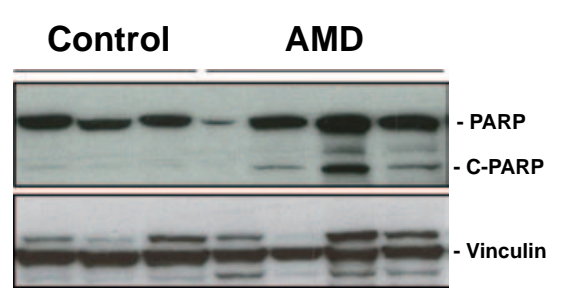

C)

OVA15

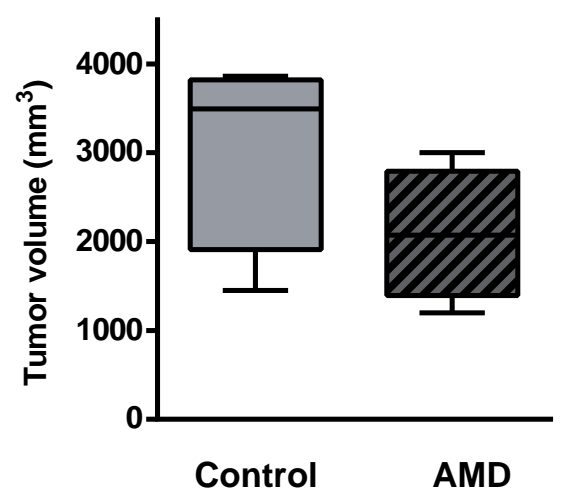

F)

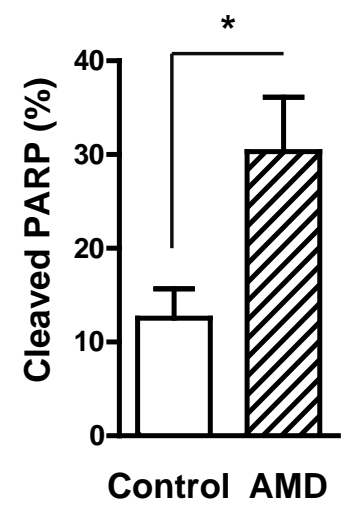


Figure 3

A)

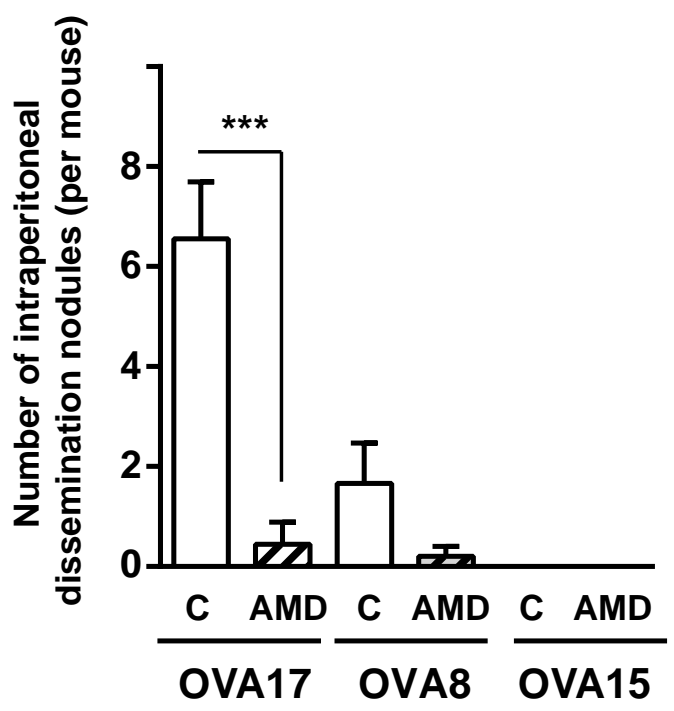

C)

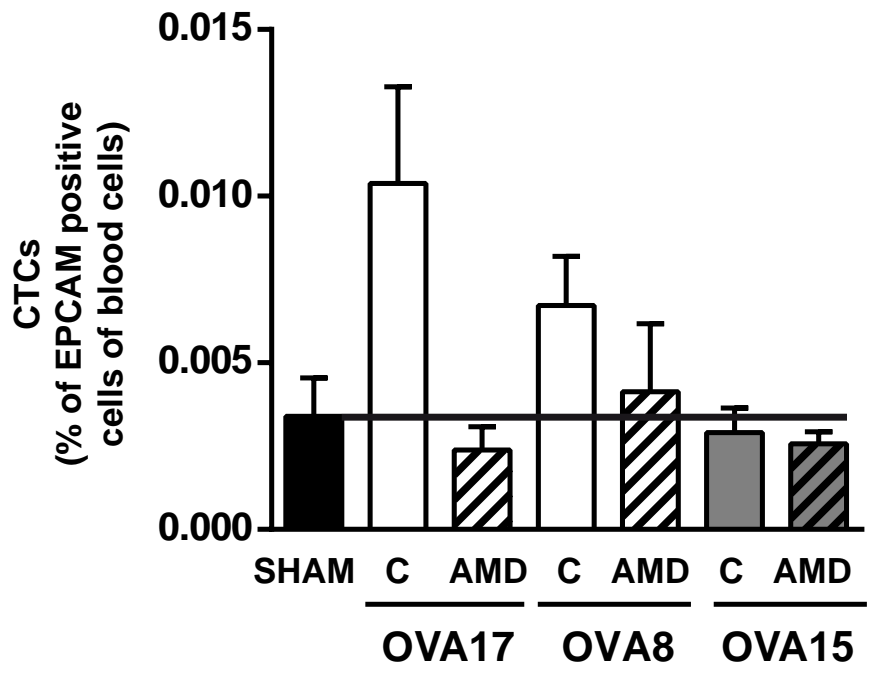

B)

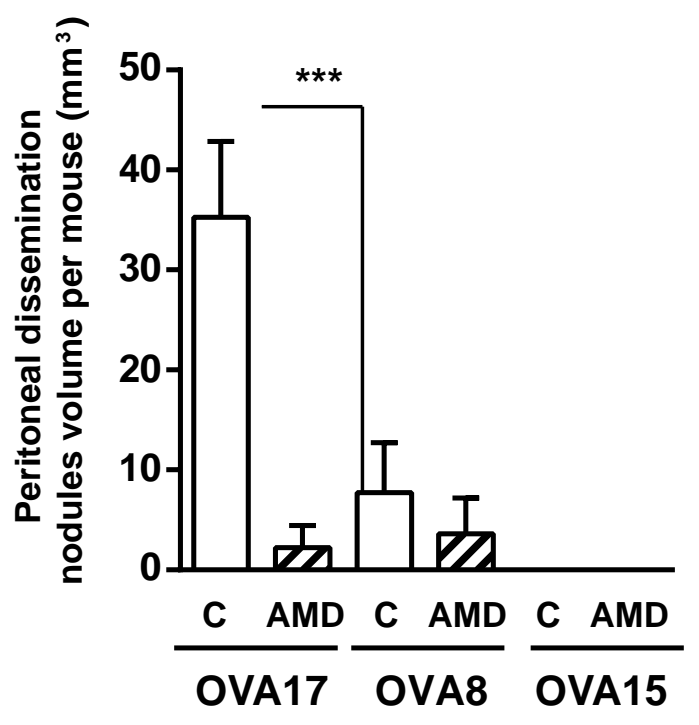

D)

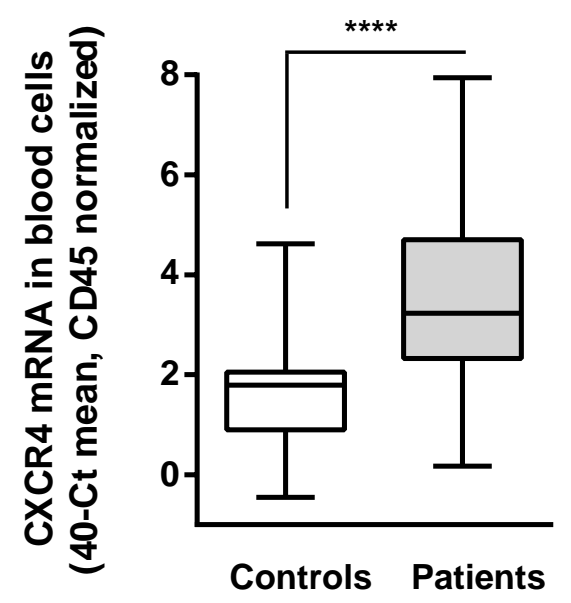


Figure 4

A)

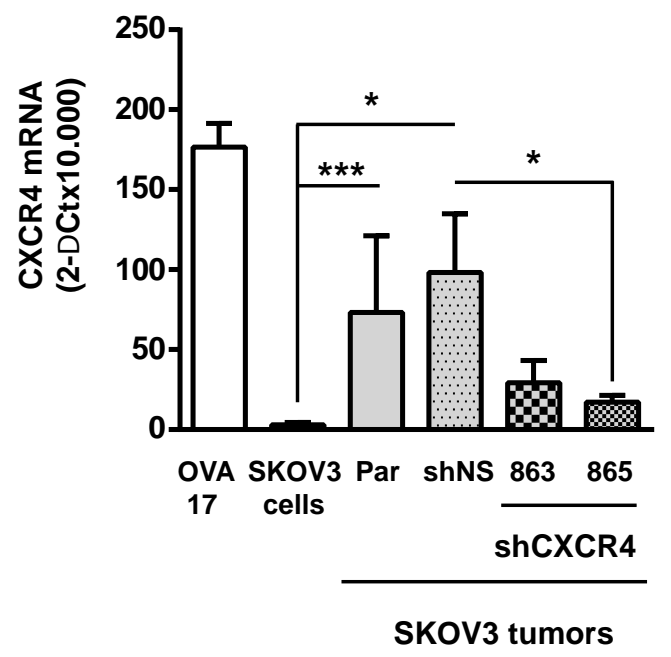

C)

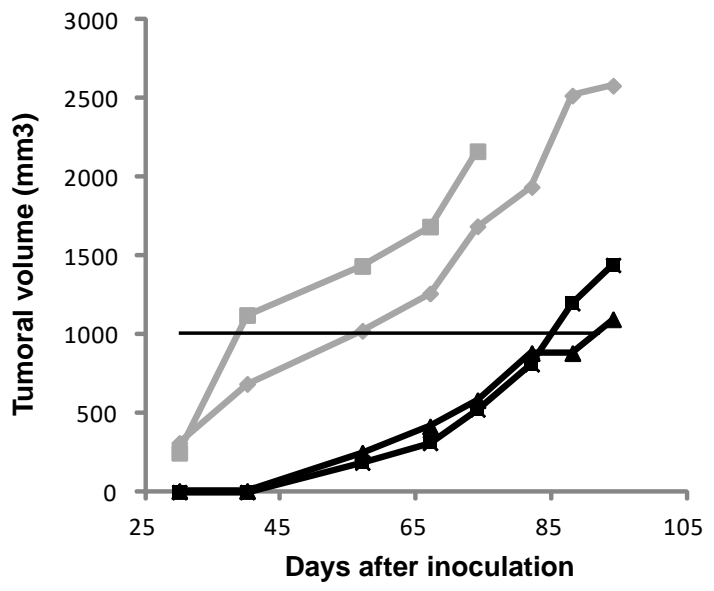

D)

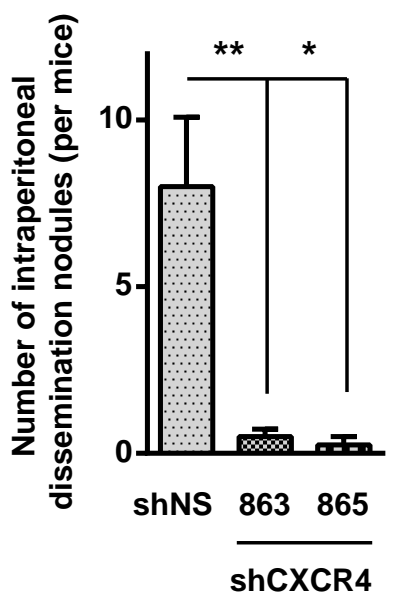

E)

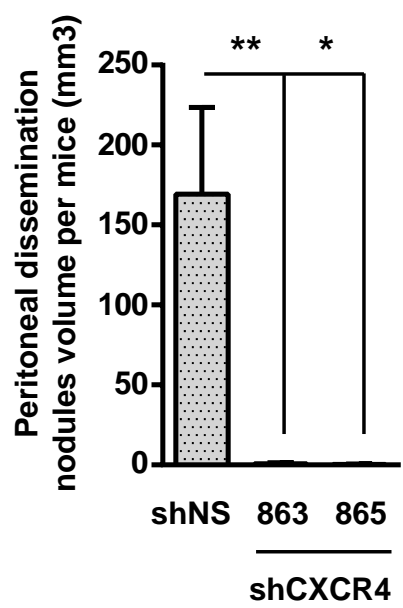

B)

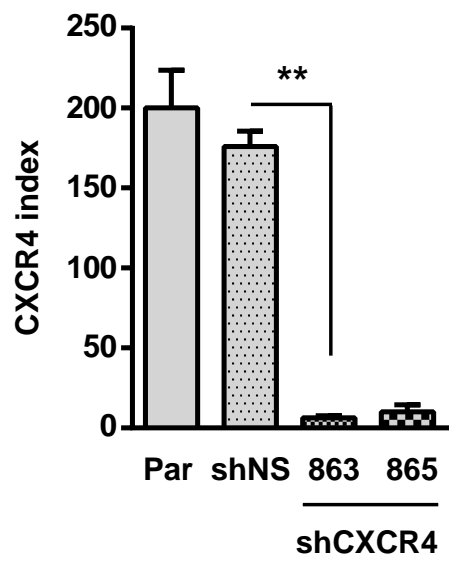

F)

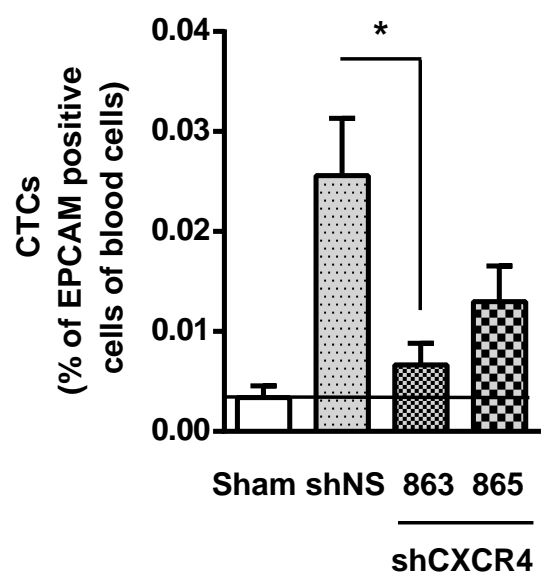


Figure 5

A)

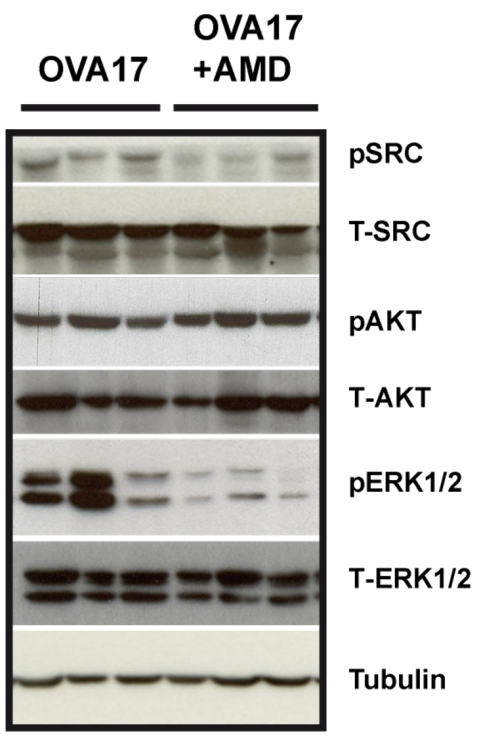

E)

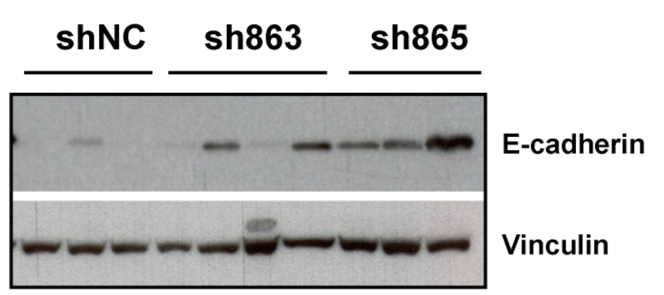

G)

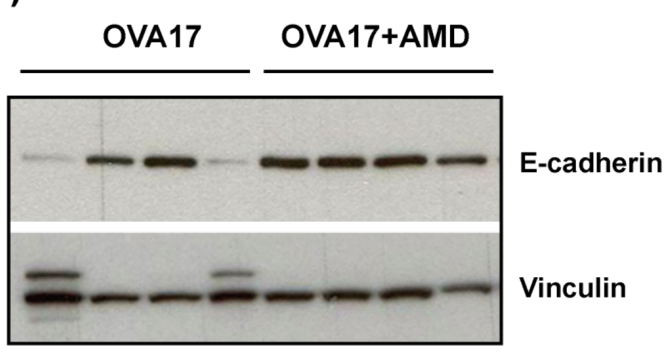

B)

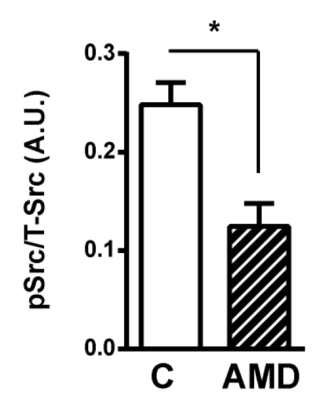

C)

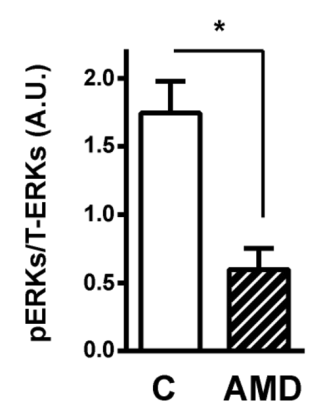

F)

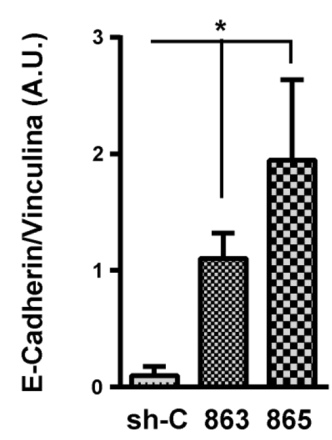

H)

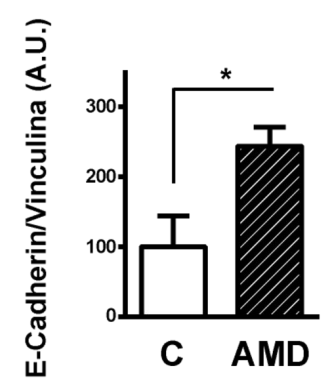

D) E-Cad Zeb1 Zeb2 Snail

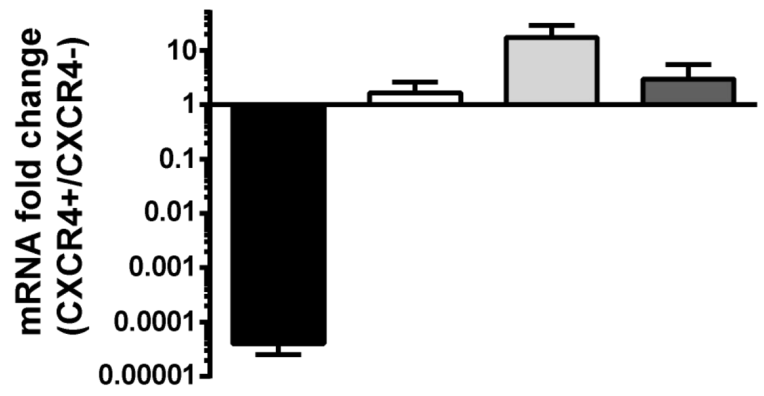

I)

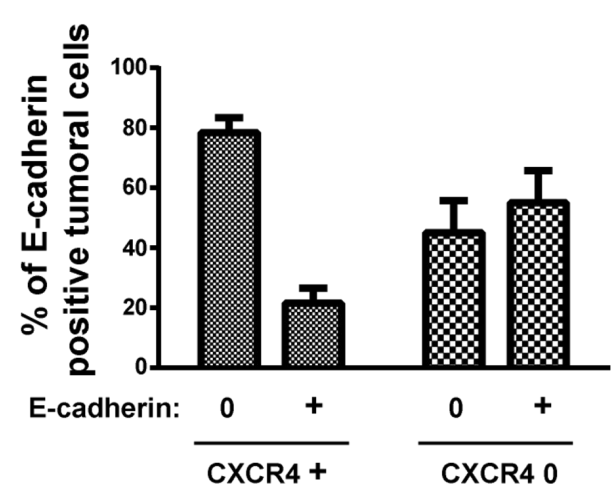

